\title{
The use of lumbar epidural injection of platelet lysate for treatment of radicular pain
}

\author{
Christopher Centeno ${ }^{1,2}$, Jason Markle ${ }^{1}$, Ehren Dodson ${ }^{1,2^{*}}$ (D, lan Stemper ${ }^{2}$, Matthew Hyzy ${ }^{1}$, Christopher Williams ${ }^{1}$ \\ and Michael Freeman ${ }^{3}$
}

\begin{abstract}
Background: Epidural steroid injections (ESI) are the most common pain management procedure performed in the US, however evidence of efficacy is limited. In addition, there is early evidence that the high dose of corticosteroids used can have systemic side effects. We describe the results of a case series evaluating the use of platelet lysate (PL) epidural injections for the treatment of lumbar radicular pain as an alternative to corticosteroids.

Methods: Registry data was obtained for patients $(N=470)$ treated with PL epidural injections presenting with symptoms of lumbar radicular pain and MRI findings that were consistent with symptoms. Collected outcomes included numeric pain score (NPS), functional rating index (FRI), and a modified single assessment numeric evaluation (SANE) rating.

Results: Patients treated with PL epidurals reported significantly lower $(p<.0001)$ NPS and FRI change scores at all time points compared to baseline. Post-treatment FRI change score means exceeded the minimal clinically important difference beyond 1 month. Average modified SANE ratings showed 49.7\% improvement at 24 months post-treatment. Twenty-nine (6.3\%) patients reported mild adverse events related to treatment.

Conclusion: Patients treated with PL epidurals reported significant improvements in pain, exceeded the minimal clinically important difference (MCID) for FRI, and reported subjective improvement through 2-year follow-up. PL may be a promising substitute for corticosteroid.
\end{abstract}

Keywords: Platelet lysate, Lumbar, Radicular, Corticosteroids, Spine, Back pain, Regenerative, Steroids

\section{Background}

Lumbar epidural steroid injection (ESI) is a nonsurgical treatment for radicular pain and low back pain (LBP). The procedure typically consists of an injection combining a corticosteroid anti-inflammatory with a local anesthetic into the epidural space below the level of the conus medullaris. ESIs are the most commonly performed pain management procedures for LBP and radiculopathy or radicular pain in the United States (Cohen 2011; Manchikanti 2004). From 1994 to 2001, the number of ESIs performed in the US increased by 629\% (Deyo et al. 2009). Expenditure on ESI procedures

\footnotetext{
* Correspondence: edodson@regenexx.com

${ }^{1}$ Centeno-Schultz Clinic, Broomfield, CO 80021, USA

${ }^{2}$ Regenexx, LLC, Des Moines, IA 50321, USA

Full list of author information is available at the end of the article
}

has increased commensurately (Deyo et al. 2009; Manchikanti 2004). In 2012, the cost of ESIs exceeded $\$ 100$ billion in the US (Wilkinson and Cohen 2012).

Despite the popularity of the procedure with pain management specialists and other clinicians, there is no clear evidence demonstrating the efficacy of ESI as a means of improving function, decreasing disability, or reducing spine surgery rates (Airaksinen et al. 2006; Armon et al. 2007; Deyo et al. 2009). Although some authors have reported mild to moderate short-term symptomatic improvement with ESI, long-term follow-up studies have not demonstrated a persisting benefit (Arden et al. 2005; Koes et al. 1995; Wilson-MacDonald et al. 2005). Indeed, a number of randomized placebo-controlled trials have concluded there is little difference in efficacy between ESI and placebo injections with saline (Deyo et al. 2009; 
Karppinen et al. 2001). It is also postulated the positive effects seen with ESI are more likely a result of the anesthetic component of the injection or the effect of diluting or displacing local inflammatory or noxious cytokines, rather than the anti-inflammatory action of the steroid (Manchikanti et al. 2015).

The therapeutic use of corticosteroids (used in high doses in ESI) is potentially associated with a multitude of adverse effects that can disrupt function of the endocrine, cardiovascular, musculoskeletal, gastrointestinal, dermatologic, metabolic, and nervous systems (Manchikanti 2002). Several authors have reported that repeated application of ESI can lead to significant bone loss and increased fracture risk in post-menopausal women (Kim and Hwang 2014; Mitra 2011; Bouvard et al. 2010). Other authors have demonstrated suppression of the hypothalamic-pituitary-adrenal axis persisting beyond 21 days post-ESI (Chon and Moon 2012). Reported adverse effects related to corticosteroid exposure include transient systolic blood pressure elevation and increased postprandial glucose levels that persisted for a longer time in diabetic versus non-diabetic patients (Kim and Hwang 2014; Younes et al. 2007).

Given the potential for negative health effects, as well as the equivocal evidence of efficacy for ESI, it is reasonable to seek out alternative non-operative treatments for lumbar radiculopathy or radicular pain. Regenerative therapies are a class of treatments, designed to replace, regenerate, or mitigate catabolism of damaged tissue. The therapies often utilize autologous blood products such as platelet rich plasma (PRP). PRP is the concentration of platelets obtained after isolation from the peripheral whole blood sample. PRP is used to promote healing and believed to act via the release of growth factors (GF) found inside platelets (Mautner and Kneer 2014). The inherent nature of autologous PRP has been shown in multiple studies to be safe, while the use of PRP for common orthopedic conditions such tendinopathies and osteoarthritis in peripheral joints has been promising (Sandrey 2014; Anitua et al. 2014). Published clinical evidence for PRP use in spinal conditions has been limited to lumbar spinal facet syndrome and intervertebral disc pathology (Wu et al. 2017; Tuakli-Wosornu et al. 2016; Kirchner and Anitua 2016; Akeda et al. 2017). Our own early clinical experience of injecting PRP into the intervertebral disc as part of a mesenchymal stem cell (MSC) procedure demonstrated no safety issues, which is consistent with reports from other authors (Levi et al. 2015; Centeno et al. 2011). Utilizing PRP for the treatment of radiculopathy has been limited to a small pilot study with $N=10$, that found gradual improvements sustained through 3 months follow-up (Bhatia and Chopra 2016).
Platelet lysate (PL) is created by lysing platelets and removing the cell debris, resulting in a GF-rich injectate, devoid of other platelet material (Doucet et al. 2005). PRP and PL have both shown promise for clinical use in the treatment of orthopedic injuries over the past decade (Centeno 2014; Lopez-Vidriero et al. 2010; Randelli et al. 2014). Of note, there have been several studies documenting the beneficial effects of PRP for the treatment of peripheral neuropathy (e.g. carpal tunnel syndrome) and peripheral nerve regeneration after injury (Abbasipour-Dalivand et al. 2015; Anjayani et al. 2014; Elgazzar et al. 2008; Hibner et al. 2012; Kuffler et al. 2011a; Malahias et al. 2015). As an alternative to ESI for lumbar radiculopathy or radicular pain, PL may be preferable to $\mathrm{PRP}$, as the former does not carry the potential for platelet adhesion and aggregation, which increases the risk of vascular occlusion (Wybier 2008). Given that several tragic cases of ischemic spinal cord lesions have been reported via particulate steroid occlusion of radicular arteries, scrutiny concerning the embolic nature of injectates is warranted (Pountos et al. 2016).

It has been widely reported that PL has the ability to promote the proliferation of various cell types including MSCs (Burnouf et al. 2016). Human serum (a significant component of PL) contains many different antiinflammatory proteins including alpha-2-macroglobulin, interleukin receptor antagonist, and tissue inhibitor of metalloproteinase (Chen et al. 2014; Villeneuve et al. 2009). While the use of PL injected into the epidural space for the treatment of LBP and radicular pain has not been described previously in the literature, given its inherent anti-inflammatory and nerve repair potential, it may serve as an alternative to traditional ESI.

In the present study, we describe the analysis of patient tracking data from a prospective, multi-site registry. The data consist of outcomes and complications of the treatment of a consecutive sample of patients with lumbar radicular pain who underwent lumbar epidural PL injections and entered a registry. We believe this to be the first reported patient experience of the use of platelet products in the epidural space to treat lumbar radicular pain.

\section{Methods}

Patients enrolled into a treatment registry designed to track patient safety and treatment outcomes. An Institutional Review Board (HHS OHRP \#IRB00002637) approved the registry data protocol. All patients (or their guardians if they were under 18 years of age) enrolled in the registry underwent an informed consent process before entering the registry. Enrolled patients were prospectively followed using an electronic system, ClinCapture software (Clinovo Clinical Data Solutions, 
Sunnyvale, California) that generates automated preand post-treatment questionnaires for evaluation at 1 , $3,6,12,18,24$ months, and annually thereafter.

The registry protocol was as follows: patients were sent outcome and complications questionnaires. Up to five attempts were made at each time point to contact patients. If the patient failed to respond, that time point was lost to follow-up. Patients were exited from the registry at their request or if they elected for definitive surgery. For adverse events (AEs), broad tickler questions were asked to elicit a complaint. To enhance surveillance, all treating physicians were asked to report any complications related to the procedure. Any reported AEs were adjudicated by the treating physician and classified by relatedness and severity. For more details, please see the $\mathrm{AE}$ adjudication methodology already reported in prior publications (Centeno et al. 2011; Centeno et al. 2010; Centeno et al. 2016).

The data for the present investigation was culled from the patient registry database, representing 20 physicians at 13 outpatient interventional pain clinics treating patients with PL epidurals from November 2008 to August 2015. This sample included consecutive patients presenting with complaints of lumbar pain and radiating symptoms into their lower extremity. All patients were diagnosed with lumbar radicular pain based on history, physical exams, and MRI findings consistent with the diagnosis. Concurrent explanatory diagnoses included intervertebral disc derangements (e.g. herniation, protrusion, extrusion, etc.) and foraminal and/or central canal stenosis. The only inclusion criteria was that the patient have lumbar radicular pain in need of treatment, while exclusion criteria was based on medical conditions that would preclude an epidural injection route (e.g. coagulopathy, local infection at injection site, septicemia, pregnancy, and neurological disorders such as multiple sclerosis). Injection timing and frequency was based on clinical indication and was not controlled. Outcome measures obtained at each time point were a Numeric Pain Score (NPS), a modified Single Assessment Numeric Evaluation rating (SANE), and the Functional Rating Index (FRI).

NPS is a recognized way to quantify pain similar to a segmented version of the visual analogue scale, in which a whole number is selected to reflect the intensity of pain where $0=$ no pain and $10=$ worst possible pain (Katz and Melzack 1999; Hawker et al. 2011). The modified SANE rating asked patients what percent difference had they seen compared to their condition prior to the procedure from $-100 \%$ worsened to $100 \%$ improved. This is a more rigorous version of the validated SANE metric which only allows responses from $0 \%$ to $100 \%$ (i.e. cannot determine if a patient reported a worse condition due to the procedure) (Williams et al. 1999; Shelbourne et al. 2012). The
FRI instrument contains 10 items that assess pain and function focusing on activities of daily living that gauge patient disability. A higher score on FRI is indicative of more dysfunction with 0 representing functional independence with no limitations and 100 corresponding to severe disability (Feise and Michael Menke 2001).

Data for patients' reported scores for each post-injection time point were analyzed for the three outcome measures. The differences between scores at pre- and post-treatment time points were calculated as change scores.

\section{Procedure description}

Two weeks prior to undergoing the PL epidural procedure, patients were restricted from the use of corticosteroids and non-steroidal anti-inflammatory drugs. The rationale for the restriction is based on the concern for the inhibitory effect of such drugs on healing potential (Bondesen et al. 2004). On either the day of or day prior to the procedure, patients presented to the clinic for a venous blood draw, wherein approximately $60 \mathrm{~mL}$ of heparinized venous blood was collected. The heparinized venous blood was processed by hand in a sterile ISO-5 class biologic safety cabinet. No commercial automated systems designed to produce platelet rich plasma were used. To prepare the PL, whole blood was prepped via centrifugation at $200 \mathrm{~g}$ for $10 \mathrm{~min}$ to separate plasma from red blood cells. The resultant liquid lying above the concentrated solids (supernatant) was red blood cell / white blood cell poor and platelet rich. This $3-10 \mathrm{~cm}^{3}$ supernatant was extracted via pipette. Volume of supernatant varies based on patient's hematocrit levels. All samples were then placed in a $-80^{\circ}$ Celsius freezer for 5-10 min followed by thaw. This resulting blood product was then re-centrifuged to pellet any remaining platelet bodies, and the supernatant was extracted and sent to bedside for use. If the procedure was scheduled for the following day, the sample was placed in $-20^{\circ}$ freezer overnight and then re-thawed prior to use.

Accurate needle placement into the epidural space was accomplished utilizing $\mathrm{C}$-arm fluoroscopy. Patients were treated with either a transforaminal or interlaminar epidural with the injection route determined by the examination and review of imaging. Detailed injection procedures have been previously described (Cohen 2011; Wilkinson and Cohen 2012). Once the needle was placed in the final target location and confirmed with fluoroscopy, Iodixanol (Visipaque, NDC\# 0407-2223-06) radiographic contrast was injected to confirm flow into the epidural space. The final injectate consisted of PL $50 \%$ by volume, $4 \%$ lidocaine (NDC\# 0409-4283-01) at 25\% by volume, and compounded preservative free $100-200 \mathrm{ng} / \mathrm{ml}$ hydrocortisone (obtained via different compounding pharmacies) at 25\% by volume. For transforaminal and interlaminar injections, 3-5 $\mathrm{cm}^{3}$ volume was injected. PL injectate was filtered at 
the time of injection with a $0.22-\mu \mathrm{m}$ filter (Millex-GP, Millipore express PES membrane) to remove all remaining lysed platelet membrane debris. The nanogram amount of hydrocortisone used with PL is one million times less than what is commonly used for ESI injections and is used for its anti-inflammatory properties at levels similar to endogenous glucocorticoids (Jung et al. 2014).

Post-treatment, the patients were given instructions to participate in activity as tolerated. All patients were encouraged to participate in physical therapy, though this was not required nor controlled. A physical therapy prescription was provided at the patient's request.

\section{Statistical analysis}

Baseline characteristics were described using the mean and standard deviation for continuous variables. These included patient demographic information (age, body mass index (BMI) and gender), and self-reported scores (NPS, modified SANE Rating and FRI). Changes in the FRI and NPS scores were assessed using dependent two-group t-tests between the baseline and each posttreatment score. Subjects with missing scores were excluded from respective analyses. Differences between post-treatment time points were examined using a oneway analysis of variance (ANOVA) followed by posthoc Tukey if warranted. All analyses were performed retrospectively utilizing $\mathrm{R}$, a software environment for statistical computing and graphics, version 3.2.2.

\section{Results}

The selection process for the patient population is shown in Fig. 1. Briefly, 1920 patients joined the registry tracking spine procedures. Of these, 859 received a spine procedure as a secondary injection to other diagnoses. Of the remaining 1061 patients, 399 received injections in the cervical spine, lumbar facets, lumbar ligaments, or peripheral joints, and were therefore removed. An additional 191 patients failed to provide follow-up data, resulting in final patient population of 470. This population consisted of $265(56.4 \%)$ males and 205 (43.6\%) females. Additional demographics are provided in Table 1 . From the final sample, 325 were treated by 4 physicians at one site; the remaining 145 were treated at 11 other sites by 16 different physicians. Of the 470 patients, 273 (58\%) had a single injection, 85 (18\%) had two injections, 52 (11\%) had three injections, and $60(13 \%)$ patients had four injections, each at separate visits.

\section{Adverse events}

Of the 470 patients, $44(9.4 \%)$ reported a complaint. Of those patients, 29 (6.2\% of overall population) were adjudicated by the attending physician as an adverse event

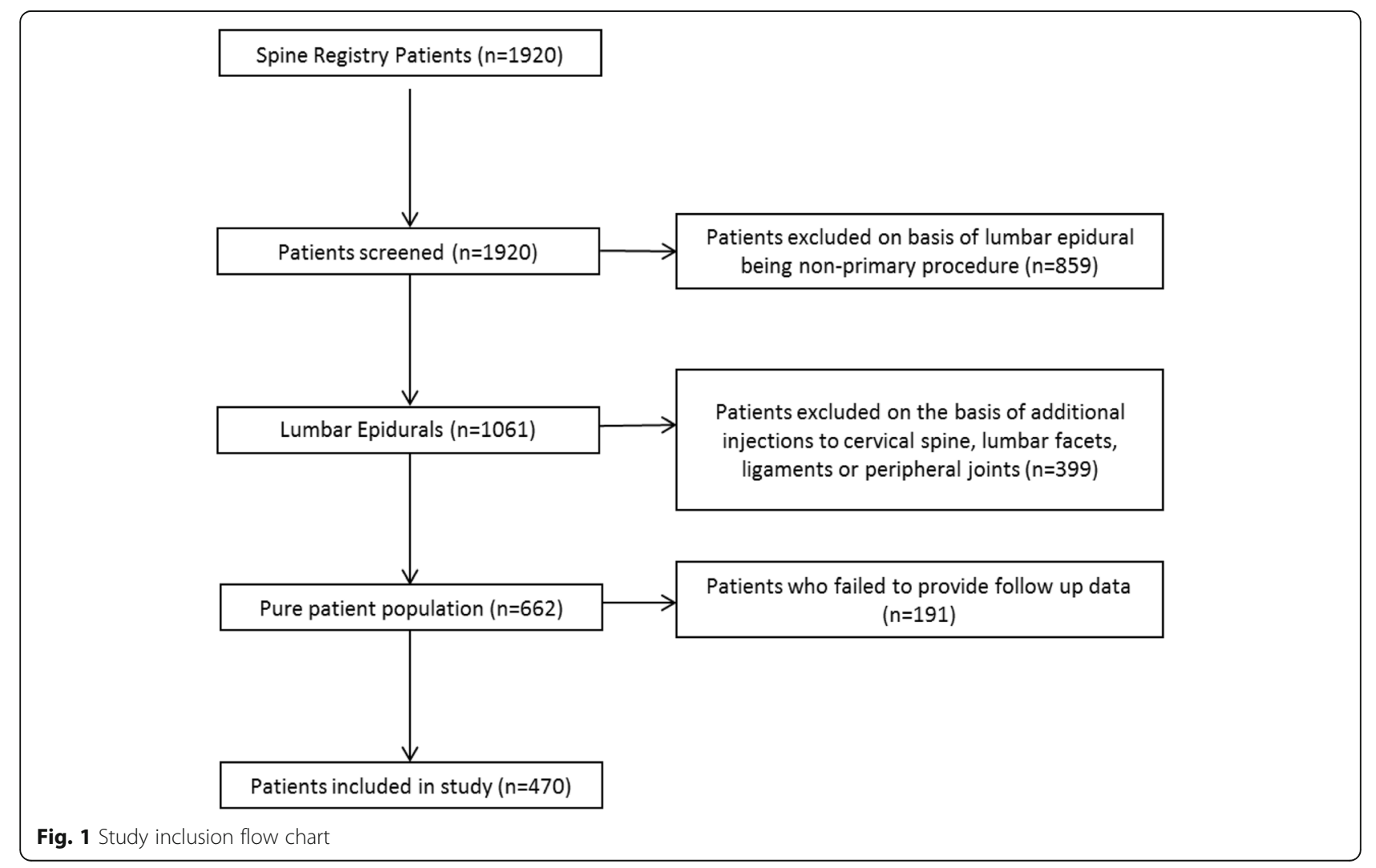


Table 1 Demographic information

\begin{tabular}{lllll}
\hline Variable & N & Mean (SD) & Min & Max \\
\hline Age & 470 & $53.6(13.5)$ & 16 & 91 \\
BMI & 307 & $26.5(4.5)$ & 18.3 & 41.5 \\
Males & $265(56.4 \%)$ & & & \\
Females & $205(43.6 \%)$ & & & \\
\hline
\end{tabular}

Number of patients, mean and standard deviation included in each calculated variable with ranges for age and $\mathrm{BMI}$

$B M I$ body mass index, $N$ number, SD standard deviation

(AE) related to the procedure. Of those 29 patients, 4 reported AEs at multiple time points, with the majority being pain related. For example, 23 patients $(79.3 \%$ of the AEs) reported pain related events, with a 2 patients reporting more than 1 event (pain $(N=18)$, inflammation $(N=1)$, soreness $(N=2)$, muscle tightening $(N=1)$, stiffness $(\mathrm{N}=2)$ and/or numbness $(N=1)$. Other AEs were categorized as dural puncture related (i.e. nausea, vomiting with positional headaches and lightheadedness, $N=3$ ) or skin reactions (redness or swelling, $\mathrm{N}=3$ ), which accounted for $20.7 \%$ of the total AEs. See Fig. 2. Of the three patients who reported symptoms consistent with dural puncture, two resolved with conservative care and the third resolved after receiving an autologous blood patch. No serious adverse events (infection, paralysis or neurologic deficit) were reported. Eleven patients (2.3\%) reported that they elected for lumbar surgery: five within 5-7 months postinjection, two at 1-year, two at 18-months and two at 2years. Over $93 \%$ of patients reported no AEs related to the procedure. All adverse events were self-limiting and resolved by $1-6$ months.

\section{Numeric pain score}

Sixty-four percent of patients $(N=303)$ provided baseline NPS data, and those without baseline data were excluded from the respective analysis to limit missing data bias. Average baseline NPS was 5.1 ( $\mathrm{SD}=2.4)$. The change in scores from pre- to post-treatment was calculated for each patient at each time point. Change score averages ranged from 1.6-2.4, with each time point being statistically lower $(p<.0001)$ than baseline (Fig. 3). Table 2 notes how NPS averages incrementally decreased from 1 through 24 months. An ANOVA showed NPS changed across post-treatment time points $(p<.005)$. Post-hoc Tukey confirmed 18 month and 24 month NPS were significantly lower than 1 month ( $p<.05$ and $p<.01$ respectively), as well as 24 month scores were significantly lower than 3 month scores $(p<.05)$.

\section{Modified SANE rating}

Of the 470 patients, 438 (93\%) provided modified SANE rating responses. The patients' last recorded responses averaged $42.4 \%$ improvement at an average of 16.6 months post-treatment. Modified SANE score means increased incrementally from $35.9 \%(\mathrm{SD}=34.2)$ at 1 month to $49.7 \%(\mathrm{SD}=42.1)$ at 24 months. A oneway ANOVA showed modified SANE scores differed significantly across time points $(p<.005)$. Post-hoc Tukey showed significantly higher modified SANE scores between 1 month and both the 18 and 24 month posttreatment time points $(p<.05)$. Scores trended toward a significant difference between the 3 month and 24 month time point $(p=.07)$. Figure 4 displays the percentage of patients self-reporting improvement, no change, or a worse condition after receiving treatment. Improvement (modified SANE > 0) was noted in $72.7-77.1 \%$ of patients across the post-treatment time points. No change was reported in $17.4-25.8 \%$, and a worse condition in $1.6-6.4 \%$ of patients. Detailed modified SANE ratings are characterized in Table 2.

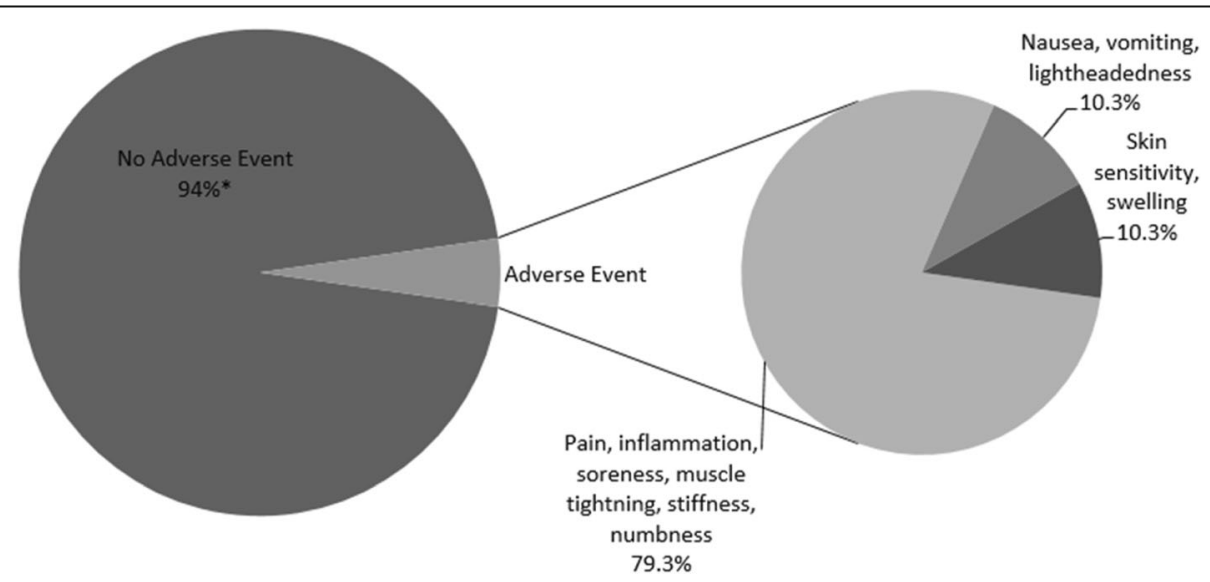

Fig. 2 Adverse events reported. Adverse events (AE) related to the procedure were reported by $6.2 \%$ of patients. Of those $79.3 \%$ were categorized as pain related, $10.3 \%$ were dural puncture-related and $10.3 \%$ were skin reactions. ${ }^{*}$ The percentage of patients without an AE related to the procedure 


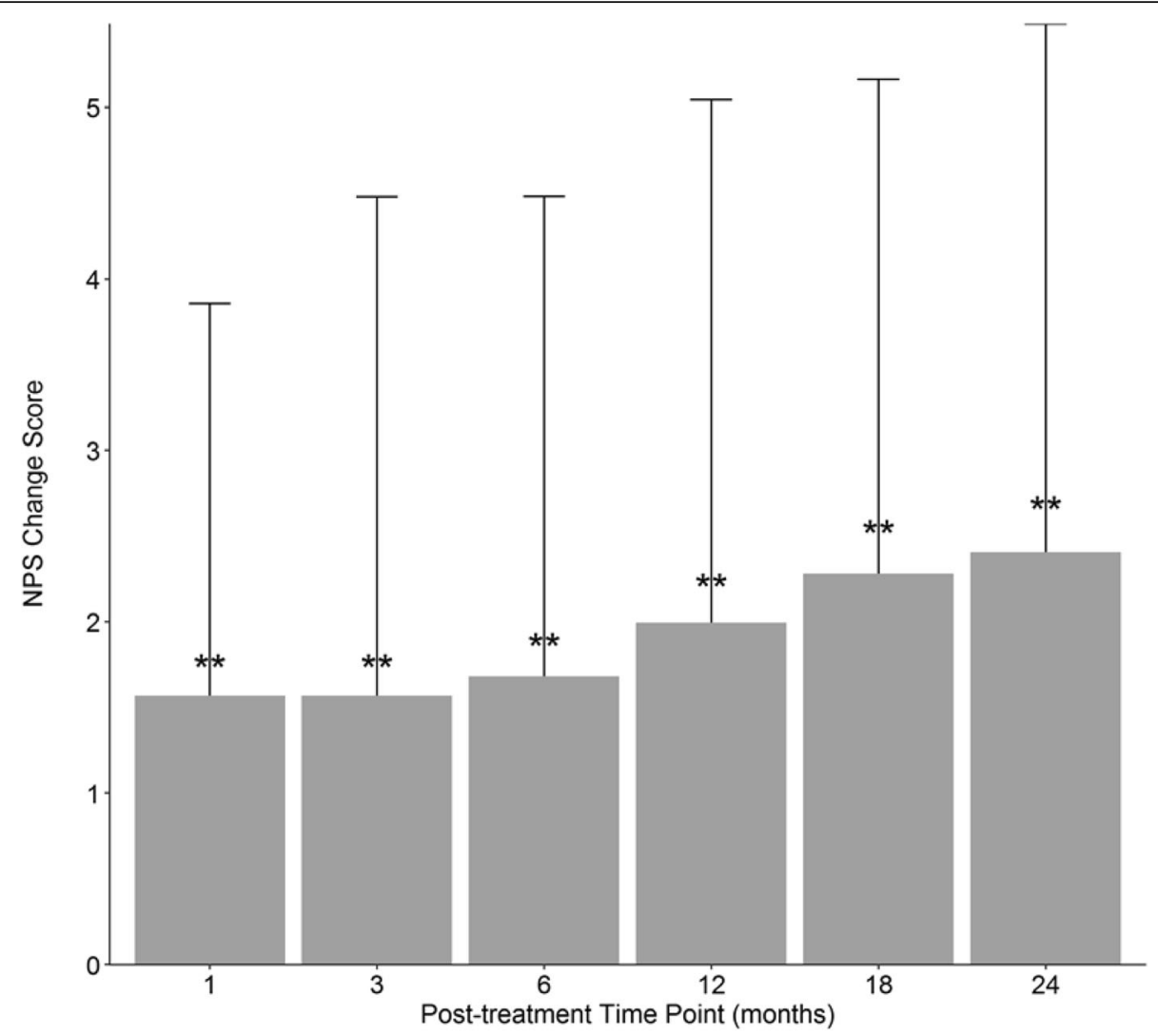

Fig. 3 NPS average change scores. Numeric pain score (NPS) average change from baseline to post-treatment with standard deviation. Number of patients reporting at each time point: 1-month $(N=139)$; 3-month $(N=192)$; 6 -month $(N=181)$; 12 -month $(N=174)$; 18 -month $(N=143)$; 24-month $(N=126)$. Statistical comparisons are to baseline. ${ }^{* *} p<.0001$

\section{Functional rating index}

We obtained baseline functional rating index scores for 239 (51\%) patients included in this analysis. Average baseline FRI score was $52.6(\mathrm{SD}=20.6)$. The change in scores from pre- to post-treatment was calculated for each patient at each time point. The average change score at each time point was significantly different from baseline $(p<.0001)$, with the average change score ranging from 8.4 to 19 (Fig. 5). The minimal clinically important

Table 2 Clinical outcomes for modified SANE, NPS and FRI

\begin{tabular}{llll}
\hline Time point & Modified SANE & NPS & FRI \\
\hline Baseline & n/a & $5.1(303)$ & $52.6(239)$ \\
1-month & $35.9(128)$ & $3.6(139)$ & $45.0(111)$ \\
3-month & $39.4(219)$ & $3.4(192)$ & $42.5(144)$ \\
6-month & $42.2(239)$ & $3.2(181)$ & $39.7(146)$ \\
12-month & $46.1(245)$ & $3.0(174)$ & $37.2(136)$ \\
18-month & $48.6(210)$ & $2.7(143)$ & $34.3(114)$ \\
24-month & $49.7(198)$ & $2.5(126)$ & $31.7(100)$ \\
\hline
\end{tabular}

Mean (number of patients) of modified SANE rating, NPS and FRI at each time point

FRI Functional Rating Index, NPS Numeric Pain Score, SANE Single Assessment Numeric Evaluation difference (MCID) for FRI is 9 points, and was met or exceeded at all time points beyond 1 month (Childs and Piva 2005). Table 2 displays the incremental decrease in average FRI scores at each time point. An ANOVA showed scores differed between post-treatment time points $(p<.0001)$. Post-hoc Tukey showed 12 month scores were mildly lower than 1 month scores $(p=.052)$. Eighteen month scores were significantly lower than 1 month scores $(p<.005)$ and 3 month scores $(p<.05)$. Scores at 24 months were significantly lower than 1 month scores $(p<.0005), 3$ month scores $(p<.005)$ and 6 month scores $(p<.05)$.

\section{Discussion}

Registry based data revealed PL epidural injections showed promise as an alternative treatment for lumbar radicular pain. Patients reported significantly less pain after treatment, maintained through 24 months. Self-reported modified SANE rating above baseline was demonstrated in the majority of patients with data $(>72 \%)$ treated with PL at every time point, which was sustained through the 24 month follow-up period. Based on FRI change scores, function increased (indicated by scores decreasing) after 


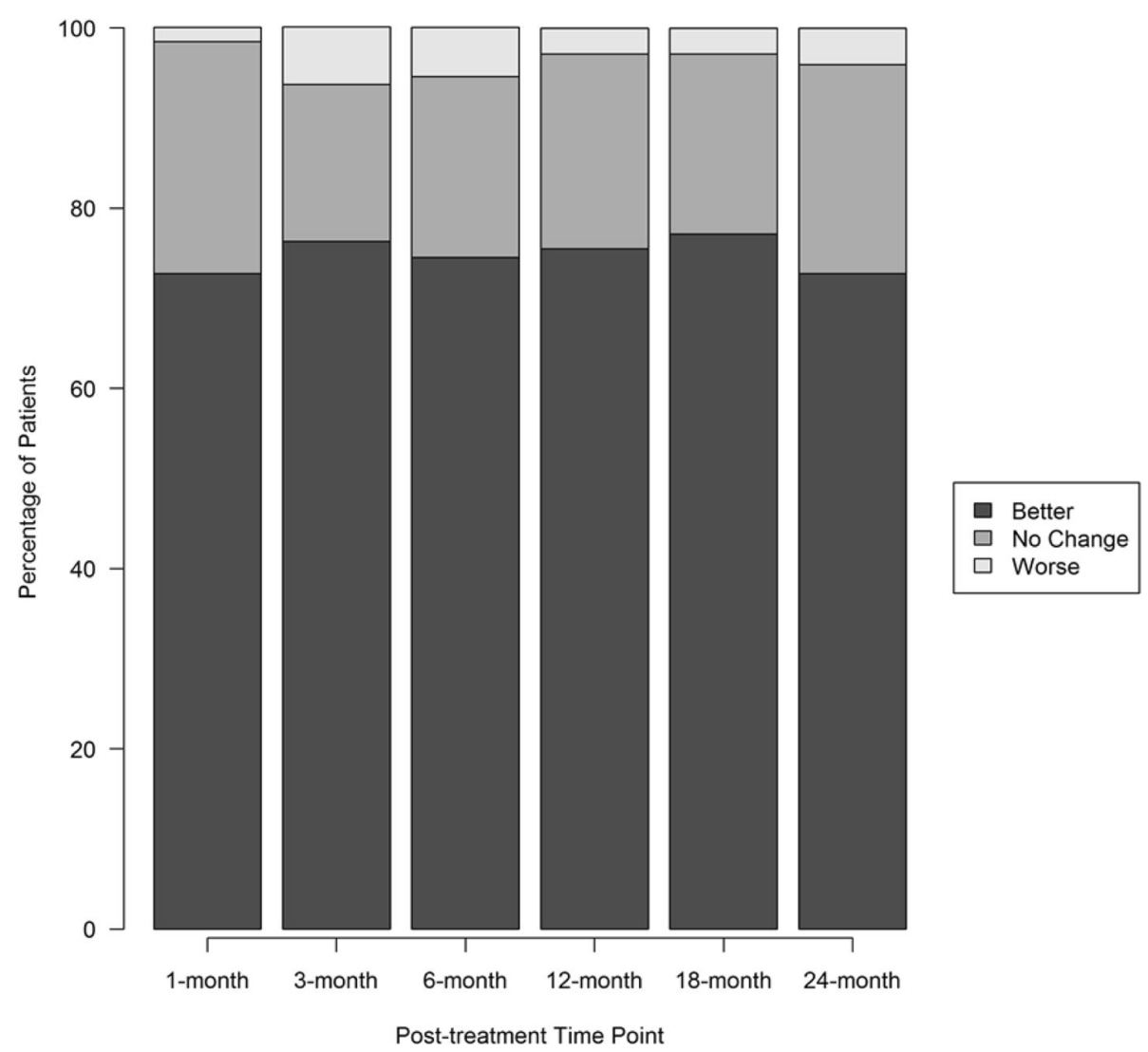

Fig. 4 Modified SANE ratings tier plot. The percent of total patients at each post-treatment time point reporting feeling better (modified SANE $>0$ ), no change (modified SANE $=0$ ), or worse (modified SANE $<0)$. Patients reporting at each time point: 1 - month $(N=128) ; 3-$ month $(N=211)$, 6 -month $(N=216)$, 12-month $(N=203)$, 18-month $(N=153)$, 24-month $(N=129)$

treatment with PL, with average change scores exceeding the minimal clinically important difference for all time points beyond 1 month post-treatment. The significant difference in pain and function at later time points compared to scores at 1,3 , and even 6 months may suggest that a continued effect of the PL occurs over time.

Of the 470 patients, none reported any serious adverse events (life-threatening infections, hospitalizations, permanent neurologic deficit, or a complication that required significant care to mitigate) with the majority of AEs related to post-treatment pain that was self-limiting (79.3\% of AEs). Other complications such as dural leaks were not more common than the expected rate for traditional ESIs (Epstein 2013). Rare adverse events included reports of lightheadedness, nausea, and vomiting. These may have resulted from a larger peripheral blood draw inducing a hypovolemic state in a minority of patients. In addition, eleven $(<3 \%)$ of all patients opted for surgery after receiving treatment. In the current study, patients treated with PL epidurals improved their function and pain significantly from baseline at both short term $(1,3$ and 6 months) and long term (12, 18 and 24 months) time points. The finding that patients reported clinically significant functional changes through 2 years is important to highlight. Previous ESI studies have shown a mild to moderate short-term benefit that was not sustained over long-term follow-ups, however, this comparison may or may not be applicable due to differences in study methodology (Arden et al. 2005; Koes et al. 1995; Wilson-MacDonald et al. 2005).

The properties of the growth factors and cytokines in PL may explain these findings, including enhancing nerve repair, improving blood supply, or reducing inflammation. These include transforming growth factor-beta (TGF- $\beta$ ), insulin-like growth factor-1 (IGF-1), vascular endothelial growth factor (VEGF), platelet-derived growth factors (PDGF), and others. Any or all of these acting individually or in conjunction may have accounted for the observed effects. For example, several GFs present in platelets have been identified as critical for nerve repair. PDGF stimulate the release of neurotrophic factors, which promote the regeneration of peripheral nerves (Monje et al. 2009). PDGF receptors are upregulated on axons and Schwan cells in injured nerves (Yamazaki et al. 2009). IGF-1 directly binds to IGF-1 receptors to stimulate the first step in fatty acid synthesis for re-myelination. The simultaneous application 


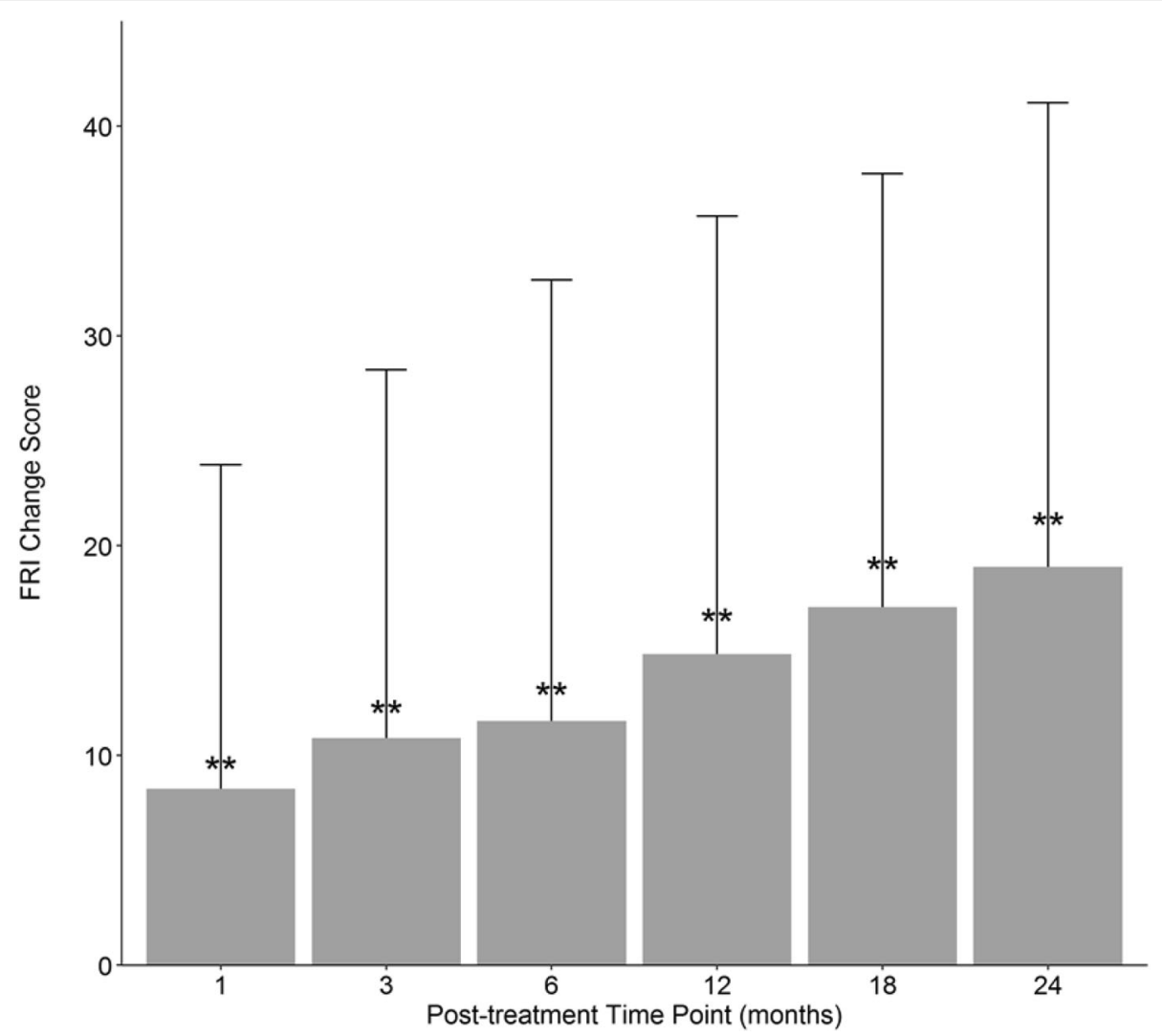

Fig. $5 \mathrm{FRI}$ average change scores. Functional rating index (FRI) averaged change in scores from baseline at each post-treatment time point with standard deviation. The number of patients reporting at each time point: 1-month $(N=111)$; 3-month $(N=144)$, 6-month $(N=146)$, 12-month $(N=136)$, 18-month $(N=114), 24-$ month $(N=100) .{ }^{* *} p<.0001$

of PDGF and IGF-1 can induce more rapid regeneration than either of them individually (Oudega et al. 1997; Liang et al. 2007). VEGF induces angiogenesis, a critical step that precedes axon regeneration in animal models (Hoke et al. 2001). TGF- $\beta$ can reactivate long-term denervated Schwann cells, triggering synthesis and release of neurotrophic factors, and thereby promote axonal regeneration (Johnson et al. 2008; Feng and Ko 2008). Kuffler and colleagues have shown in both animal models and case reports that the application of PRP can significantly improve peripheral nerve axon regeneration with gaps varying from 3 to $12 \mathrm{~cm}$ (Kuffler 2014; Kuffler et al. 2011b).

The procedure used in this study is quite different from more commonly used platelet rich plasma therapy, which was not used due to potential safety concerns regarding platelet aggregation. During epidurals, inadvertent vascular injections have been estimated at $8-12 \%$ (Epstein 2013). Coalescing platelets could lead to vascular occlusion and result in end-arterial capillary occlusion, similar to the proposed risks for particulate steroid injections (Wybier 2008). To eliminate this potential risk, PL was filtered through a $0.22 \mu \mathrm{m}$ filter to eliminate the lysed platelet membranes prior to injection.
While the clinical use of PL compared to traditional PRP for orthopedic conditions has not been previously described, the effect of PL on human cells in vitro is well known. PL use for cell culture is described extensively in the literature and may provide some insight into its potential ability to stimulate local progenitor cells (Burnouf et al. 2016). Schallamoser et al. and several others have shown the benefits of culturing and expanding mesenchymal stromal cells (MSCs) in PL (Reinisch et al. 2007; Schallmoser et al. 2007). Overall, the clinical effectiveness of PL vs traditional PRP should be equivocal, the decision to use PL for epidural injection was based off the safety profile and a direct comparison would not be advisable given the potential risks of vascular occlusion with the use of PRP in the epidural space.

Limitations of the current study include lack of a control group and randomization, missing data, multiple components of the injectate, lack of strict post-treatment controls, and potential patient bias. This study did not include an untreated control group or randomization of subjects, hence placebo effects cannot be ruled out. The outcome data collected in the registry was missing various endpoints for patients, though multiple attempts were 
made to collect outcomes for each time point. Although this is an inherent problem with registry data, this platform allows us to prospectively monitor longterm outcomes in a large population of patients. In addition, the treatment injectate contained platelet lysate, local anesthetic, and a nanogram dose of corticosteroid, any of which may have produced the clinical effect seen, either alone or in combination. However, the volume of local anesthetic and corticosteroid is dramatically less than what has been compared for epidural therapeutic purposes, which leads us to believe that it is the PL producing the clinical outcomes in this sample of patients (Manchikanti et al. 2012). Post-treatment rehabilitation was not controlled, which could have led to differences in therapeutic effects. In addition, one cannot rule out potential bias introduced from patients paying for this therapy as part of their medical care.

\section{Conclusion}

This is the first publication highlighting the clinical use of platelet lysate injected into the lumbar epidural space for the treatment of lumbar radicular pain. The analysis of the registry data shows promise for use of this therapy to reduce pain and improve function. We observed no additional risk beyond traditional steroid epidurals. While there is a need for randomized controlled trials to determine efficacy, these results highlight a potentially promising alternative to reduce the deleterious effects of high dose steroids on patients, as well as offer a possible non-surgical alternative for patients with lumbar radicular pain.

\section{Abbreviations \\ AE: Adverse events; ANOVA: Analysis of variance; BMI: Body mass index; ESI: Epidural steroid injection; FRI: Functional rating index; GF: Growth factors; IGF-1: Insulin-like growth factors-1; LBP: Low back pain; MCID: Minimal clinically important difference; MRI: Magnetic resonance imaging; MSC: mesenchymal stem cell; NPS: Numeric pain score; PDGF: Platelet derived growth factors; PL: Platelet lysate; PRP: Platelet rich plasma.; SANE: Single assessment numeric evaluation; TGF- $\beta$ : Transforming growth- beta; VEGF: Vascular endothelial growth factors}

\section{Acknowledgments}

Not applicable.

\section{Availability of data and materials}

The datasets generated or analyzed during this study are not publically available to ensure patient privacy and $\mathrm{PHI}$ is not compromised, but are available from the corresponding author on reasonable request.

\section{Authors' contributions}

CC, JM, ED, IS and MF participated in study design. CC, JM, ED, IS, CW, MH and MF all participated in data analysis and interpretation and the preparation of the manuscript. All authors read and approved the final manuscript.

\section{Funding}

This research was funded by Regenexx, LLC and the Centeno-Schultz Clinic

\section{Ethics approval and consent to participate}

Patients provided verbal and written consent. The International Cellular Medicine Society provided IRB oversight and approval (OHRP Registration
\#IRB00002637). Trial registration number: NCT03011398; A Clinical Registry of Orthobiologics Procedures.

\section{Consent for publication}

All patients provided consent for publication.

\section{Competing interests}

CC is a shareholder and CMO of Regenexx, LLC and president and owner of the Centeno-Schultz Clinic. JM, ED, IS, CW, MH and MF have declared no competing interests.

\section{Publisher's Note}

Springer Nature remains neutral with regard to jurisdictional claims in published maps and institutional affiliations.

\section{Author details}

${ }^{1}$ Centeno-Schultz Clinic, Broomfield, CO 80021, USA. ${ }^{2}$ Regenexx, LLC, Des Moines, IA 50321, USA. ${ }^{3}$ CAPHRI School of Public Health and Primary Care, Maastricht University, Maastricht, Netherlands.

Received: 23 August 2017 Accepted: 25 October 2017

Published online: 25 November 2017

\section{References}

Abbasipour-Dalivand S, Mohammadi R, Mohammadi V (2015) Effects of local Administration of Platelet Rich Plasma on functional recovery after bridging sciatic nerve defect using silicone rubber chamber; an experimental study. Bulletin of emergency and. Trauma 3(1):1-7

Airaksinen O, Brox Jl, Cedraschi C, Hildebrandt J, Klaber-Moffett J, Kovacs F, Mannion AF, Reis S, Staal JB, Ursin H, Zanoli G, Pain CBWGoGfCLB, On behalf of the CBWGoGfCLBP (2006) Chapter 4 European guidelines for the management of chronic nonspecific low back pain European Spine Journal 15 (S2):s192-s300. doi:10.1007/s00586-006-1072-1

Akeda K, Ohishi K, Masuda K, Bae WC, Takegami N, Yamada J, Nakamura T, Sakakibara T, Kasai Y, Sudo A (2017) Intradiscal injection of autologous platelet-rich plasma Releasate to treat Discogenic low back pain: a preliminary clinical trial. Asian Spine journal 11(3):380-389. doi:10.4184/asj. 2017.11.3.380

Anitua E, Sanchez M, Aguirre JJ, Prado R, Padilla S, Orive G (2014) Efficacy and safety of plasma rich in growth factors intra-articular infiltrations in the treatment of knee osteoarthritis. Arthroscopy : the journal of arthroscopic \& related surgery : official publication of the Arthroscopy Association of North America and the International Arthroscopy Association 30 (8):1006-1017. doi: 10.1016/j.arthro.2014.05.021

Anjayani S, Wirohadidjojo YW, Adam AM, Suwandi D, Seweng A, Amiruddin MD (2014) Sensory improvement of leprosy peripheral neuropathy in patients treated with perineural injection of platelet-rich plasma. Int J Dermatol 53(1): 109-113. doi:10.1111/ijd.12162

Arden NK, Price C, Reading I, Stubbing J, Hazelgrove J, Dunne C, Michel M, Rogers P, Cooper C, Group WS (2005) A multicentre randomized controlled trial of epidural corticosteroid injections for sciatica: the WEST study. Rheumatology (Oxford, England) 44(11):1399-1406. doi:10.1093/ rheumatology/kei028

Armon C, Argoff CE, Samuels J, Backonja M-M, Therapeutics, Technology Assessment Subcommittee of the American Academy of N (2007) Assessment: use of epidural steroid injections to treat radicular lumbosacral pain: report of the therapeutics and technology assessment Subcommittee of the American Academy of neurology. Neurology 68 (10):723-729. doi:10. 1212/01.wnl.0000256734.34238.e7

Bhatia R, Chopra G (2016) Efficacy of Platelet Rich Plasma via Lumbar Epidural Route in Chronic Prolapsed Intervertebral Disc Patients-A Pilot Study. Journal of clinical and diagnostic research : JCDR 10 (9):Uc05-uc07. doi:10.7860/jcdr/ 2016/21863.8482

Bondesen BA, Mills ST, Kegley KM, Pavlath GK (2004) The COX-2 pathway is essential during early stages of skeletal muscle regeneration. Am J Physiol Cell Physiol 287(2):C475-C483. doi:10.1152/ajpcell.00088.2004

Bouvard B, Legrand E, Audran M, Chappard D (2010) Glucocorticoid-induced osteoporosis: a review. Clin Rev Bone Miner Metab 8(1):15-26. doi:10.1007/ s12018-009-9051-9 
Burnouf T, Strunk D, Koh MB, Schallmoser K (2016) Human platelet lysate: replacing fetal bovine serum as a gold standard for human cell propagation? Biomaterials 76:371-387. doi:10.1016/j.biomaterials.2015.10.065

Centeno CJ (2014) Clinical challenges and opportunities of mesenchymal stem cells in musculoskeletal medicine. PM R 6(1):70-77. doi:10.1016/j.pmrj.2013.08.612

Centeno CJ, Al-Sayegh H, Freeman MD, Smith J, Murrell WD, Bubnov R (2016) A multi-center analysis of adverse events among two thousand, three hundred and seventy two adult patients undergoing adult autologous stem cell therapy for orthopaedic conditions. Int Orthop 40(8):1755-1765. doi:10.1007/ s00264-016-3162-y

Centeno CJ, Schultz JR, Cheever M, Freeman M, Faulkner S, Robinson B, Hanson R (2011) Safety and complications reporting update on the re-implantation of culture-expanded mesenchymal stem cells using autologous platelet lysate technique. Current stem cell research \& therapy 6(4):368-378

Centeno CJ, Schultz JR, Cheever M, Robinson B, Freeman M, Marasco W (2010) Safety and complications reporting on the re-implantation of cultureexpanded mesenchymal stem cells using autologous platelet lysate technique. Curr Stem Cell Res Ther 5(1):81

Chen X, Kong X, Zhang Z, Chen W, Chen J, Li H, Cao W, Ge Y, Fang S (2014) Alpha-2-macroglobulin as a radioprotective agent: a review. Chinese journal of cancer research $=$ Chung-kuo yen cheng yen chiu 26(5):611-621. doi:10. 3978/j.issn.1000-9604.2014.09.04

Childs JD, Piva SR (2005) Psychometric properties of the functional rating index in patients with low back pain. Eur Spine J 14(10):1008-1012. doi:10.1007/ s00586-005-0900-Z

Chon JY, Moon HS (2012) Salivary cortisol concentration changes after epidural steroid injection. Pain Physician 15(6):461-466

Cohen SP (2011) Epidural steroid injections for low back pain. BMJ (Clinical research ed) 343(7823):d5310

Deyo RA, Mirza SK, Turner JA, Martin Bl (2009) Overtreating chronic back pain: time to back off? J Am Board Fam Med 22(1):62

Doucet C, Ernou I, Zhang Y, Llense JR, Begot L, Holy X, Lataillade JJ (2005) Platelet lysates promote mesenchymal stem cell expansion: a safety substitute for animal serum in cell-based therapy applications. J Cell Physiol 205(2):228-236. doi:10.1002/jcp.20391

Elgazzar RF, Mutabagani MA, Abdelaal SE, Sadakah AA (2008) Platelet rich plasma may enhance peripheral nerve regeneration after cyanoacrylate reanastomosis: a controlled blind study on rats. Int J Oral Maxillofac Surg 37(8):748-755. doi:10.1016/j.jom.2008.05.010

Epstein NE (2013) The risks of epidural and transforaminal steroid injections in the spine: commentary and a comprehensive review of the literature. Surg Neurol Int 4(Suppl 2):S74-S93. doi:10.4103/2152-7806.109446

Feise RJ, Michael Menke J (2001) Functional rating index: a new valid and reliable instrument to measure the magnitude of clinical change in spinal conditions. Spine (Phila Pa 1976) 26(1):78-86 discussion 87

Feng Z, Ko CP (2008) Schwann cells promote synaptogenesis at the neuromuscular junction via transforming growth factor-beta1. J Neurosci 28(39):9599-9609. doi:10.1523/jneurosci.2589-08.2008

Hawker GA, Mian S, Kendzerska T, French M (2011) Measures of adult pain: visual analog scale for pain (VAS pain), numeric rating scale for pain (NRS pain), McGill pain questionnaire (MPQ), short-form McGill pain questionnaire (SF$M P Q)$, chronic pain grade scale (CPGS), short Form-36 bodily pain scale (SF36 BPS), and measure of intermittent and constant osteoarthritis pain (ICOAP). Arthritis Care Res 63(Suppl 11):S240-S252. doi:10.1002/acr.20543

Hibner M, Castellanos ME, Drachman D, Balducci J (2012) Repeat operation for treatment of persistent pudendal nerve entrapment after pudendal neurolysis. J Minim Invasive Gynecol 19(3):325-330. doi:10.1016/j.jmig.2011.12.022

Hoke A, Sun HS, Gordon T, Zochodne DW (2001) Do denervated peripheral nerve trunks become ischemic? The impact of chronic denervation on vasa nervorum. Exp Neurol 172(2):398-406. doi:10.1006/exnr.2001.7808

Johnson EO, Charchanti A, Soucacos PN (2008) Nerve repair: experimental and clinical evaluation of neurotrophic factors in peripheral nerve regeneration. Injury 39(Suppl 3):S37-S42. doi:10.1016/j.injury.2008.06.015

Jung C, Greco S, Nguyen HH, Ho JT, Lewis JG, Torpy DJ, Inder WJ (2014) Plasma, salivary and urinary cortisol levels following physiological and stress doses of hydrocortisone in normal volunteers. BMC Endocr Disord 14(1):1-10. doi:10. 1186/1472-6823-14-91

Karppinen J, Malmivaara A, Kurunlahti M, Kyllönen E, Pienimäki T, Nieminen P, Ohinmaa A, Tervonen O, Vanharanta H (2001) Periradicular infiltration for sciatica: a randomized controlled trial. Spine 26(9):1059-1067. doi:10.1097/ 00007632-200105010-00015
Katz J, Melzack R (1999) Measurement of pain. Surg Clin North Am 79(2):231-252 Kim S, Hwang B (2014) Relationship between bone mineral density and the frequent administration of epidural steroid injections in postmenopausal women with low back pain. Pain Research \& Management : the journal of the Canadian pain. Society 19(1):30-34

Kirchner F, Anitua E (2016) Intradiscal and intra-articular facet infiltrations with plasma rich in growth factors reduce pain in patients with chronic low back pain. J Craniovertebral Junction Spine 7(4):250-256. doi:10. 4103/0974-8237.193260

Koes BW, Scholten RJPM, Mens JMA, Bouter LM (1995) Efficacy of epidural steroid injections for low-back pain and sciatica: a systematic review of randomized clinical trials. Pain 63(3):279-288. doi:10.1016/0304-3959(95)00124-7

Kuffler DP (2014) An assessment of current techniques for inducing axon regeneration and neurological recovery following peripheral nerve trauma. Prog Neurobiol 116:1. doi:10.1016/j.pneurobio.2013.12.004

Kuffler DP, Reyes O, Sosa IJ, Santiago-Figueroa J (2011a) Neurological recovery across a 12-cm-long ulnar nerve gap repaired 3.25 years post trauma: case report. Neurosurgery 69(6):E1321-E1326. doi:10.1227/NEU.0b013e31822a9fd2

Kuffler DP, Reyes O, Sosa IJ, Santiago-Figueroa J (2011b) Neurological recovery across a 12-cm-long ulnar nerve gap repaired 3.25 years post trauma: case report. 69 (6):E1321

Levi D, Horn S, Tyszko S, Levin J, Hecht-Leavitt C, Walko E (2015) Intradiscal platelet-rich plasma injection for chronic Discogenic low back pain: preliminary results from a prospective trial. Pain medicine (Malden, Mass). doi:10.1093/pm/pnv053

Liang G, Cline GW, Macica CM (2007) IGF-1 stimulates de novo fatty acid biosynthesis by Schwann cells during myelination. Glia 55(6):632-641. doi:10. 1002/glia.20496

Lopez-Vidriero E, Goulding KA, Simon DA, Sanchez M, Johnson DH (2010) The use of platelet-rich plasma in arthroscopy and sports medicine: optimizing the healing environment. Arthroscopy 26(2):269-278. doi:10.1016/j.arthro. 2009.11.015

Malahias MA, Johnson EO, Babis GC, Nikolaou VS (2015) Single injection of platelet-rich plasma as a novel treatment of carpal tunnel syndrome. Neural Regen Res 10(11):1856-1859. doi:10.4103/1673-5374.165322

Manchikanti L (2002) Role of neuraxial steroids in interventional pain management. Pain physician 5(2):182

Manchikanti $L$ (2004) The growth of interventional pain management in the new millennium: a critical analysis of utilization in the Medicare population. Pain Physician 7(4):465-482

Manchikanti L, Cash KA, McManus CD, Pampati V, Fellows B (2012) Results of 2-year follow-up of a randomized, double-blind, controlled trial of fluoroscopic caudal epidural injections in central spinal stenosis. Pain Physician 15(5):371-384

Manchikanti L, Pampati V, Benyamin RM, Boswell MV (2015) Analysis of efficacy differences between caudal and lumbar interlaminar epidural injections in chronic lumbar axial discogenic pain: local anesthetic alone vs. local combined with. Steroids 12(3):214

Mautner K, Kneer L (2014) Treatment of tendinopathies with platelet-rich plasma. Phys Med Rehabil Clin N Am 25(4):865-880. doi:10.1016/j.pmr.2014.06.008

Mitra R (2011) Adverse effects of corticosteroids on bone metabolism: a review. PM\&R 3(5):466-471. doi:10.1016/j.pmrj.2011.02.017

Monje PV, Rendon S, Athauda G, Bates M, Wood PM, Bunge MB (2009) Nonantagonistic relationship between mitogenic factors and CAMP in adult Schwann cell re-differentiation. Glia 57(9):947-961. doi:10.1002/glia.20819

Oudega M, XM X, Guenard V, Kleitman N, Bunge MB (1997) A combination of insulin-like growth factor-I and platelet-derived growth factor enhances myelination but diminishes axonal regeneration into Schwann cell grafts in the adult rat spinal cord. Glia 19(3):247-258

Pountos I, Panteli M, Walters G, Bush D, Giannoudis PV (2016) Safety of epidural corticosteroid injections. Drugs in R\&D 16(1):19-34. doi:10.1007/s40268-0150119-3

Randelli P, Randelli F, Ragone V, Menon A, D'Ambrosi R, Cucchi D, Cabitza P, Banfi G (2014) Regenerative medicine in rotator cuff injuries. Biomed Res Int 2014:129515. doi:10.1155/2014/129515

Reinisch A, Bartmann C, Rohde E, Schallmoser K, Bjelic-Radisic V, Lanzer G, Linkesch W, Strunk D (2007) Humanized system to propagate cord blood-derived multipotent mesenchymal stromal cells for clinical application. 2 (4):371

Sandrey MA (2014) Autologous growth factor injections in chronic tendinopathy. J Athl Train 49(3):428-430. doi:10.4085/1062-6050-49.3.06

Schallmoser K, Bartmann C, Rohde E, Reinisch A, Kashofer K, Stadelmeyer E, Drexler C, Lanzer G, Linkesch W, Strunk D (2007) Human platelet lysate can 
replace fetal bovine serum for clinical-scale expansion of functional mesenchymal stromal cells. Transfusion 47(8):1436-1446. doi:10.1111/j.15372995.2007.01220.x

Shelbourne KD, Barnes AF, Gray T (2012) Correlation of a single assessment numeric evaluation (SANE) rating with modified Cincinnati knee rating system and IKDC subjective total scores for patients after $\mathrm{ACL}$ reconstruction or knee arthroscopy. Am J Sports Med 40(11):2487-2491. doi:10.1177/ 0363546512458576

Tuakli-Wosornu YA, Terry A, Boachie-Adjei K, Harrison JR, Gribbin CK, LaSalle EE, Nguyen JT, Solomon JL, Lutz GE (2016) Lumbar Intradiskal platelet-rich plasma (PRP) injections: a prospective, double-blind, randomized controlled study. Pm r 8(1):1-10; quiz 10. doi:10.1016/j.pmrj.2015.08.010

Villeneuve J, Block A, Le Bousse-Kerdiles MC, Lepreux S, Nurden P, Ripoche J, Nurden AT (2009) Tissue inhibitors of matrix metalloproteinases in platelets and megakaryocytes: a novel organization for these secreted proteins. Exp Hematol 37(7):849-856. doi:10.1016/j.exphem.2009.03.009

Wilkinson IM, Cohen SP (2012) Epidural steroid injections. Curr Pain Headache Rep 16(1):50-59. doi:10.1007/s11916-011-0236-9

Williams GN, Gangel TJ, Arciero RA, Uhorchak JM, Taylor DC (1999) Comparison of the single assessment numeric evaluation method and two shoulder rating scales. Outcomes measures after shoulder surgery. Am J Sports Med 27(2):214-221

Wilson-MacDonald J, Burt G, Griffin D, Glynn C (2005) Epidural steroid injection for nerve root compression. A randomised, controlled trial. J Bone Joint Surg British Vol 87(3):352-355. doi:10.1302/0301-620X.87B3.15338

Wu J, Zhou J, Liu C, Zhang J, Xiong W, Lv Y, Liu R, Wang R, Du Z, Zhang G, Liu Q (2017) A prospective study comparing platelet-rich plasma and local anesthetic (LA)/corticosteroid in intra-articular injection for the treatment of lumbar facet joint syndrome. Pain Pract 17(7):914-924. doi:10.1111/papr.12544

Wybier M (2008) Transforaminal epidural corticosteroid injections and spinal cord infarction. Joint Bone Spine 75(5):523-525. doi:10.1016/j.jbspin.2008.07.001

Yamazaki T, Sabit H, Oya T, Ishii Y, Hamashima T, Tokunaga A, Ishizawa S, Jie S, Kurashige Y, Matsushima T, Furuta I, Noguchi M, Sasahara M (2009) Activation of MAP kinases, Akt and PDGF receptors in injured peripheral nerves. Journal of the peripheral nervous system : JPNS 14(3):165-176. doi:10.1111/j.15298027.2009.00228.x

Younes M, Neffati F, Touzi M, Hassen-Zrour S, Fendri Y, Béjia I, Ben Amor A, Bergaoui N, Najjar MF (2007) Systemic effects of epidural and intra-articular glucocorticoid injections in diabetic and non-diabetic patients. Joint Bone Spine 74(5):472-476. doi:10.1016/j.jbspin.2006.10.009

\section{Submit your manuscript to a SpringerOpen ${ }^{\circ}$ journal and benefit from:}

- Convenient online submission

- Rigorous peer review

- Open access: articles freely available online

- High visibility within the field

- Retaining the copyright to your article

Submit your next manuscript at $\gg$ springeropen.com 